\title{
Synthesis of Tin Oxide Anchored on Graphene Sheets and its Performance Lithium Storage
}

\author{
Lilai Liu ${ }^{1, a^{*}}$, Peixia Yang ${ }^{2}$, Haijiao Zhang $^{3}$, Shuang $\mathrm{Li}^{3}$ and Chao Yang ${ }^{1}$ \\ ${ }^{1}$ College of Environmental and Chemical Engineering, Heilongjiang University of Science and \\ Technology, Harbin 150022 China \\ ${ }^{2}$ School of Chemical Engineering and Technology, Harbin Institute of Technology, Harbin 150001 \\ China \\ ${ }^{3}$ Engineering Institute of Graphite New Materials, Heilongjiang University of Science and \\ Technology, Harbin 150022 China \\ a Illusth@163.com
}

\begin{abstract}
Keywords: $\mathrm{SnO}_{2}$; graphene; composite; anode; lithium-ion batteries
Abstract. The $\mathrm{SnO}_{2}$ /graphene composite has been prepared by a one-step hydrothermal method. The monodisperse $\mathrm{SnO}_{2}$ nanoparticles of $\sim 3 \mathrm{~nm}$ in diameter are not only attached onto the surface of graphene sheets by anchoring with surface functional groups, but they also are encapsulated in pore channels and formed 3D electronic conductive network. The $\mathrm{SnO}_{2} /$ graphene composite exhibites excellent electr ${ }^{\circ}$ Chemical performances in lithium-ion batteries through the synergy of the monodisperse $\mathrm{SnO}_{2}$ and the high specific capacity of graphene sheets. The first discharge/charge capacity is $1843 / 1244 \mathrm{~mA} \mathrm{~h} \mathrm{~g}^{-1}$ at a current density of $100 \mathrm{~mA} \mathrm{~g}^{-1}$ and the reversible specific capacity remains $698 \mathrm{~mA} \mathrm{~h} \mathrm{~g}^{-1}$ after 100 cycles.
\end{abstract}

\section{Introduction}

Lithium-ion batteries (LIBs), as power sources for mobile communication devices, portable electronic devices and electrical vehicles, have been used widely in our life ${ }^{[1]}$. Graphite is the most widely used anode material in commercial LIBs. However it cannot qualify for high capacity batteries because its theoretical lithium storage capacity is only $372 \mathrm{mAh} \mathrm{g}^{-1}$. In order to improve the energy density of batteries, scientists have made great efforts to explore alternative anode materials with higher capacity ${ }^{[2]}$. $\mathrm{SnO}_{2}$ has attracted much attention as an alternative anode material for rechargeable LIBs. It possesses a specific capacity of $782 \mathrm{mAh} \mathrm{g}^{-1}$, which is much higher than that of traditional graphitic carbon. However a main challenge to its widespread application is the large volume expansion of $\sim 300 \%$ induced during the charge/discharge cycles ${ }^{[3]}$. To improve the stability of $\mathrm{SnO}_{2}$ electrodes and obtain better capacity retention and cycle life for $\mathrm{SnO}_{2}$, the introduction of carbonaceous materials, such as carbon nanofibers, carbon nanotubes and graphene sheets have been generally accepted due to their high electronic conductivity, excellent buffering effect and mechanical strength ${ }^{[4]}$. Graphene, a one-atom-thick planar sheet of $\mathrm{sp}^{2}$-bonded carbon with a theoretical lithium storage capacity of 744

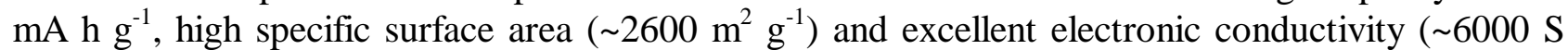
$\left.\mathrm{cm}^{-1}\right)$, has been widely studied for potential application in LIBs because of its unique properties ${ }^{[5]}$. Therefore, much research of $\mathrm{SnO}_{2}$ /graphene composites used as an anode material has been conducted. Recently, several varieties of graphene/ $\mathrm{SnO}_{2}$ nanoparticle composites have been reported as anode materials for LIBs ${ }^{[6-11]}$. These $\mathrm{SnO}_{2} /$ graphene hybrids used as anode for LIBs had different structures and exhibited excellent electr ${ }^{\circ}$ Chemical performance. However, an easy and efficient method for synthesizing $\mathrm{SnO}_{2}$ /graphene composite with excellent cycling performance for LIBs should be developed. Herein, we report a one-step hydrothermal method for synthesis of $\mathrm{SnO}_{2}$ /graphene composite with excellent electr ${ }^{\circ}$ Chemical performances as an anode material for LIBs. 


\section{Experimental}

Materials preparation. Graphene oxide (GO) was prepared by a modified Hummers method as we reported previously ${ }^{[12]}$. The obtained GO was solved in deionized water, followed by ultrasonic treatment for $60 \mathrm{~min}$. The final suspension of $\mathrm{GO}$ was controlled at $1 \mathrm{mg} \mathrm{mL} \mathrm{m}^{-1}$. The $\mathrm{SnO}_{2} / \mathrm{graphene}$ composite was prepared by a one-step hydrothermal. In a typical reaction, a certain quality of $\mathrm{SnCl}_{4} \cdot 5 \mathrm{H}_{2} \mathrm{O}$ was dissolved in $50 \mathrm{~mL}$ ethanol, and then added $\mathrm{GO}$ aqueous solution, the molar ratio of $\mathrm{SnCl}_{4} \cdot 5 \mathrm{H}_{2} \mathrm{O}$ and $\mathrm{GO}$ was $2: 1$. Adjusted $\mathrm{pH}$ value to 10 used ammonia, followed by ultrasonic treatment for $60 \mathrm{~min}$. The suspension solution was transferred to a high pressure reactor and reacted at $160{ }^{\circ} \mathrm{C}$ for $15 \mathrm{~h}$. Thus-prepared sample was washed with deionized water and ethanol for several times by centrifugation until the $\mathrm{pH}$ value of the filtered solution was 7 , and then dried at $80{ }^{\circ} \mathrm{C}$ overnight. As comparison, pure $\mathrm{SnO}_{2}$ was prepared by the same method without the addition of GO.

Materials characterizations. The structures and morphologies of the as-prepared materials were characterized by SEM (QUANTA 200F), TEM (FEI TECNAI G2 F20) and XRD (Bruker D8 Advance with $\mathrm{Cu} \mathrm{K} \alpha$ radiation) operated at $40 \mathrm{kV}$ and $40 \mathrm{~mA}$.

Electr ${ }^{\circ}$ Chemical measurements. Electr $^{\circ}$ Chemical measurements were carried out using CR2025 coin-type cells. The working electrode was prepared by coating slurries consisting of the active material, polyvinylidene fluoride and acetylene black with a weight ratio of 80:10:10 in $\mathrm{N}$-methyl-pyrrolidone solvent. The electrolyte was $1 \mathrm{M} \mathrm{LiPF}_{6}$ dissolved in a mixture of dimethyl carbonate, diethyl carbonate and ethylene carbonate (1:1:1 by weight). Galvanostatic charge-discharge curves of the cells were recorded by a Battery Testing System (Neware Electronic Co., China) at various current densities from 50 to $700 \mathrm{~mA} \mathrm{~h} \mathrm{~g}^{-1}$ with the voltage between 3.00 and $0.01 \mathrm{~V}$ versus $\mathrm{Li}^{+} / \mathrm{Li}$ at room temperature. Cyclic voltammetry $(\mathrm{CV})$ curves were measured from 0.01 to $3.00 \mathrm{~V}$ at a scanning rate of $0.1 \mathrm{mV} \mathrm{s}^{-1}$.

\section{Results and discussion}

The crystal structures of the materials were analyzed by XRD. Fig. 1 shows the XRD patterns of GO, pure $\mathrm{SnO}_{2}$ nanoparticles and $\mathrm{SnO}_{2} /$ graphene composite. The diffraction peak at $\sim 11^{\circ}$ can be attributed to (002) of the GO. The diffraction peaks of $\mathrm{SnO}_{2}$ nanoparticles are clearly distinguishable. All strong diffraction peaks are consistent with tetragonal crystalline $\mathrm{SnO}_{2}$ phase (JCPDS card no. 41-1445). No diffraction peaks of graphene and $\mathrm{GO}$ are observed in the $\mathrm{SnO}_{2}$ /graphene composite, indicating that GO is reduced to graphene, the graphene layer is exfoliated completely and the diffraction peaks of graphene are covered by those of $\mathrm{SnO}_{2}{ }^{[13]}$. This observation helps support the inference that GO is reduced in situ by bivalent tin ions.

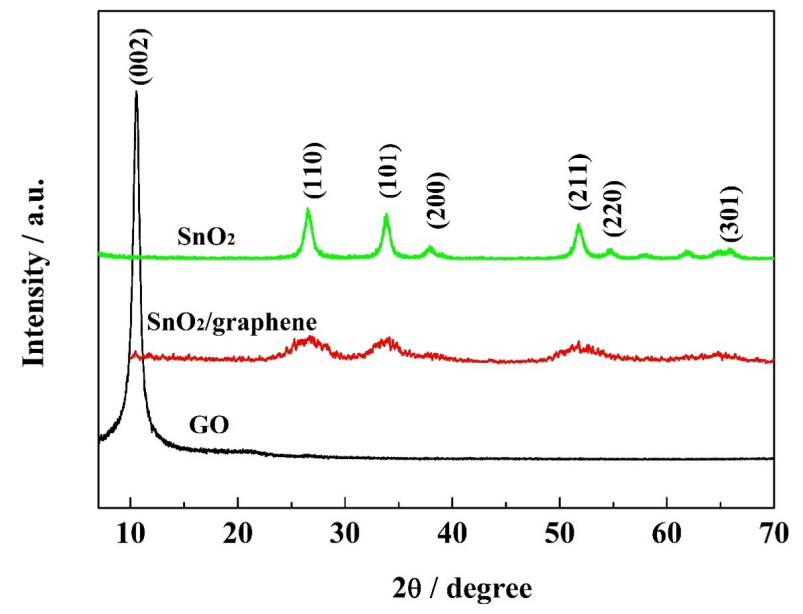

Fig. 1. XRD patterns of GO, pure $\mathrm{SnO}_{2}$ nanoparticles and $\mathrm{SnO}_{2} /$ graphene composite

The morphologies of GO, $\mathrm{SnO}_{2}$ and the $\mathrm{SnO}_{2}$ /graphene composite were observed by SEM, TEM and HRTEM. Fig. 2(a) and (b) present the SEM images of GO. It is obvious that the GO are composed of thinner transparent sheets, the area of sheets is larger, and there are many folds. The transparency after 
ultrasonic treatment likes a piece of gauze and implies that the GO sheets are only a few layers. Fig. 2(c) shows TEM image of $\mathrm{SnO}_{2}$ nanoparticles prepared by hydrothermal method, and the average particle diameter of $\mathrm{SnO}_{2}$ nanoparticles is about $13 \mathrm{~nm}$. The TEM image of $\mathrm{SnO}_{2} /$ graphene composite in Fig. 2(d) reveals that the graphene sheets is decorated with the $\mathrm{SnO}_{2}$ nanoparticles on the basal plane. The $\mathrm{SnO}_{2}$ nanoparticles are in the diameters of $\sim 3 \mathrm{~nm}$, uniformly anchored on the surface of graphene sheets, indicating that the $\mathrm{GO}$ can prevent the $\mathrm{SnO}_{2}$ nanoparticles from growing effectively. From the HRTEM images in Fig. 2(e), the interplanar distance of 0.34 can be identified as d (110) of $\mathrm{SnO}_{2}$ nanoparticles. Fig. 2(f) shows the electron diffraction pattern of $\mathrm{SnO}_{2}$ /graphene composite, the four distinct diffraction rings represent (110), (101), (200), and (210) from the rutile phase of $\mathrm{SnO}_{2}$, confirming the highly crystalline nature of $\mathrm{SnO}_{2}$ nanoparticles ${ }^{[14]}$. The smaller size of $\mathrm{SnO}_{2}$ nanoparticles and the optimized microstructure of $\mathrm{SnO}_{2}$ /graphene composite are expected to offer a better rate capability of the anode.
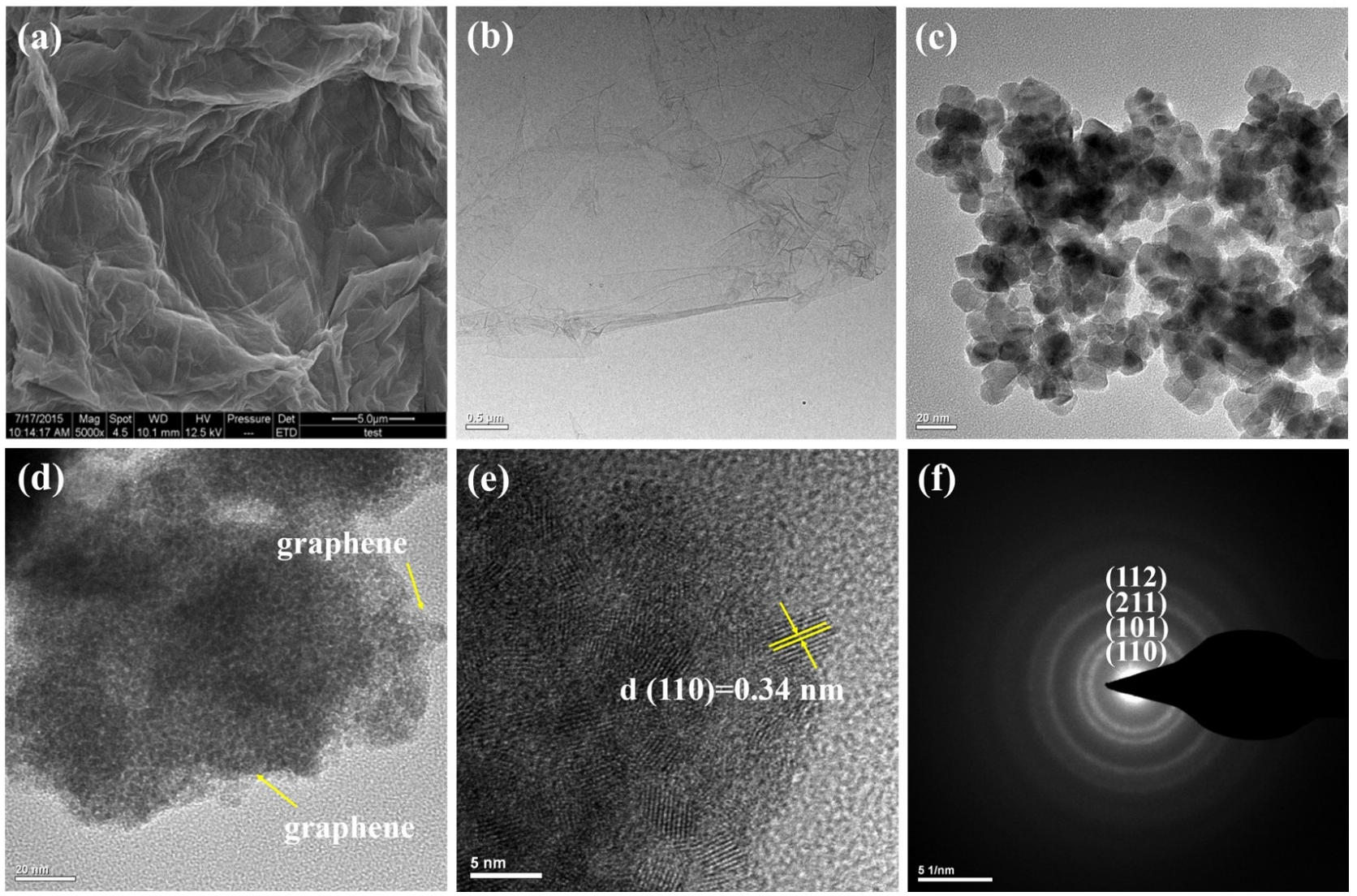

Fig. 2. SEM image of (a) GO, TEM image of (b) GO after ultrasonic treatment, TEM image

(c) $\mathrm{SnO}_{2}$ and (d) $\mathrm{SnO}_{2}$ /graphene, HRTEM images of (e) $\mathrm{SnO}_{2}$ /graphene, and

(f) Electron diffraction pattern of $\mathrm{SnO}_{2} /$ graphene

The Li-ion storage behavior of $\mathrm{SnO}_{2}$ /graphene composite was revealed by the $\mathrm{CV}$ curve and charge-discharge profiles. Fig. 3(a) shows the CV curves of the first, second and third cycles of the $\mathrm{SnO}_{2}$ /graphene composite at a scan rate of $0.1 \mathrm{mV} \mathrm{s}^{-1}$. In the first cycle, two obvious cathodic peaks appeared around 0.85 and $0.02 \mathrm{~V}$. The peak around $0.85 \mathrm{~V}$ is ascribed to the formation of the solid electrolyte interface (SEI) layers on the surface of the active material, the reduction of $\mathrm{SnO}_{2}$ to $\mathrm{Sn}$. The peak at approximately $0.02 \mathrm{~V}$ corresponds to the formation of a series of $\mathrm{Li}_{\mathrm{x}} \mathrm{Sn}$ alloys. In the first anodic $\mathrm{pr}^{\circ} \mathrm{Cess}$, there is a small peak near $0.17 \mathrm{~V}$, which can be attributed to Li intercalation into graphite to form $\mathrm{LiC}_{6}$. There are also two obvious plateaus at 0.61 and $1.28 \mathrm{~V}$, which can be ascribed to $\mathrm{Li}$ dealloying from $\mathrm{Li}_{\mathrm{x}} \mathrm{Sn}$ and the partially reversible reaction from $\mathrm{Sn}$ to $\mathrm{SnO}_{2}$, respectively. The CV curves clearly elucidated the reversible electr ${ }^{\circ}$ Chemical reactions between the lithium ions and the $\mathrm{SnO}_{2}$ /graphene composite in lithium ion batteries. Fig. 3(b) shows the 1st, 2nd and 3rd discharge and charge curves of $\mathrm{SnO}_{2}$ /graphene composite electrode at a current density of $100 \mathrm{~mA} \mathrm{~g}^{-1}$ in the voltage 
range of 3.00 0.01 V vs. $\mathrm{Li}^{+} / \mathrm{Li}$. The potential plateaus observed in the discharge curves are consistent with the $\mathrm{CV}$ results, which corresponds to a classical conversion reaction between $\mathrm{SnO}_{2}$ and $\mathrm{Li}^{+}$. In the first cycle, the discharge and charge capacities of $\mathrm{SnO}_{2} /$ graphene electrode are approximately 1843 and $1244 \mathrm{~mA} \mathrm{~h} \mathrm{~g}^{-1}$ with coulomb efficiencie of $67.5 \%$. The irreversible capacity loss in the first cycle is attributed to the decomposition of electrolyte forming SEI.
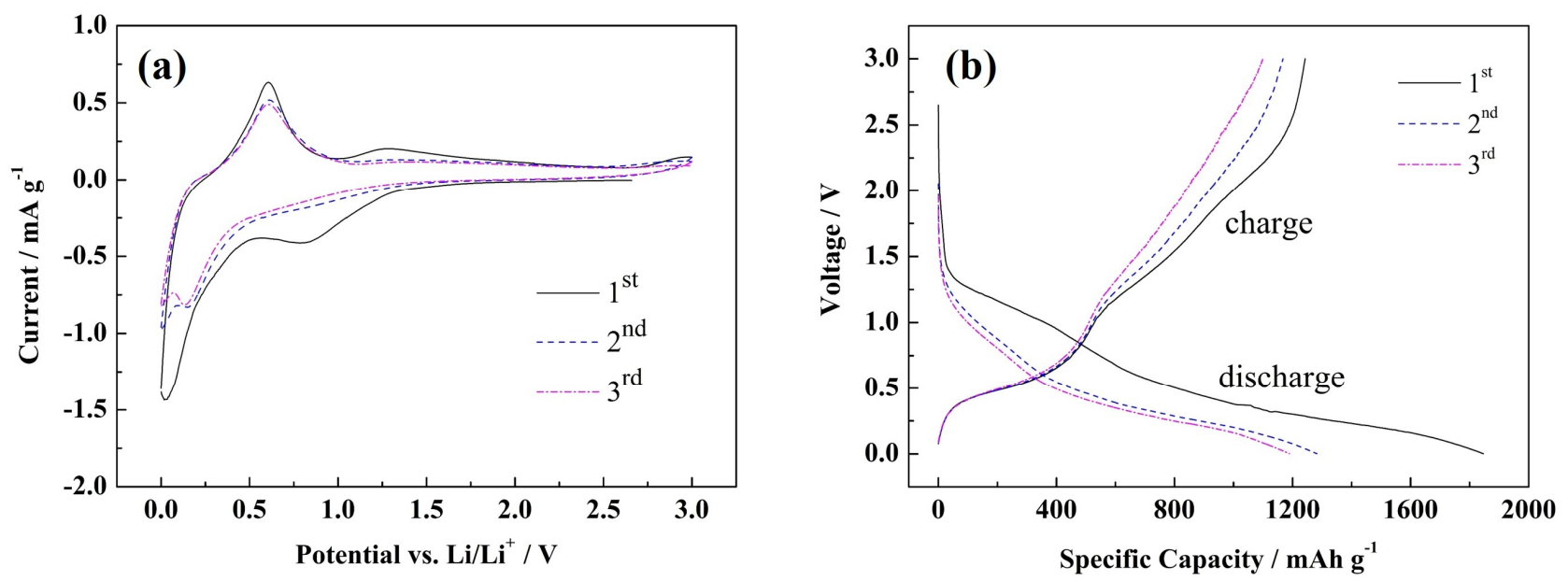

Fig. 3. (a) CV curves of different cycles of $\mathrm{SnO}_{2} /$ graphene at a scan rate of $0.1 \mathrm{mV} \mathrm{s}^{-1}$;

(b) Galvanostatic charge-discharge curves of $\mathrm{SnO}_{2} /$ graphene at a current density of $100 \mathrm{~mA} \mathrm{~g}^{-1}$

Fig. 4 shows the cycling performances and rate performance of $\mathrm{SnO}_{2} /$ graphene composite electrode. The $\mathrm{SnO}_{2}$ /graphene composite electrode demonstrates good cycling performances at different current densities, as shown in Fig. 4(a). The discharge specific capacities are 698, 654 and $587 \mathrm{~mA} \mathrm{~h} \mathrm{~g}^{-1}$ at current densities of 100,500 and $700 \mathrm{~mA} \mathrm{~g}^{-1}$ after 100 cycles, respectively. Fig. 4(b) shows the rate performance of $\mathrm{SnO}_{2} /$ graphene electrode towards different current densities $\left(50 \sim 700 \mathrm{~mA} \mathrm{~g}{ }^{-1}\right)$, and reverses back to low current density of $50 \mathrm{~mA} \mathrm{~g}^{-1}$. With the increases of current density, the reversible specific capacities of $\mathrm{SnO}_{2}$ /graphene composite electrode are 1149, 1007, 888, 743, 656, 619, 599, and $570 \mathrm{~mA} \mathrm{~h} \mathrm{~g}^{-1}$, respectively. A reversible capacity of $956 \mathrm{~mA} \mathrm{~h} \mathrm{~g}^{-1}$ is achieved after the current density back to $50 \mathrm{~mA} \mathrm{~g}^{-1}$. The good rate performance of the $\mathrm{SnO}_{2} /$ graphene composite electrode is attributed to the higher electrical conductivity and the special 2D structure of graphene, which not only greatly reduce the intrinsic ohm resistance of the composite electrode, but also facile the $\mathrm{Li}^{+}$diffusion. In addition, graphene sheets can not only provide a support for dispersing $\mathrm{SnO}_{2}$ nanoparticles and work as a highly conductive matrix for enabling good contact between them, but also can effectively prevent the volume expansion/contraction and aggregation of $\mathrm{SnO}_{2}$ nanoparticles during Li charge/discharge $\mathrm{pr}^{\circ}$ Cess. Thus, it is considered to be a promising anode material of LIBs for the electric vehicles.
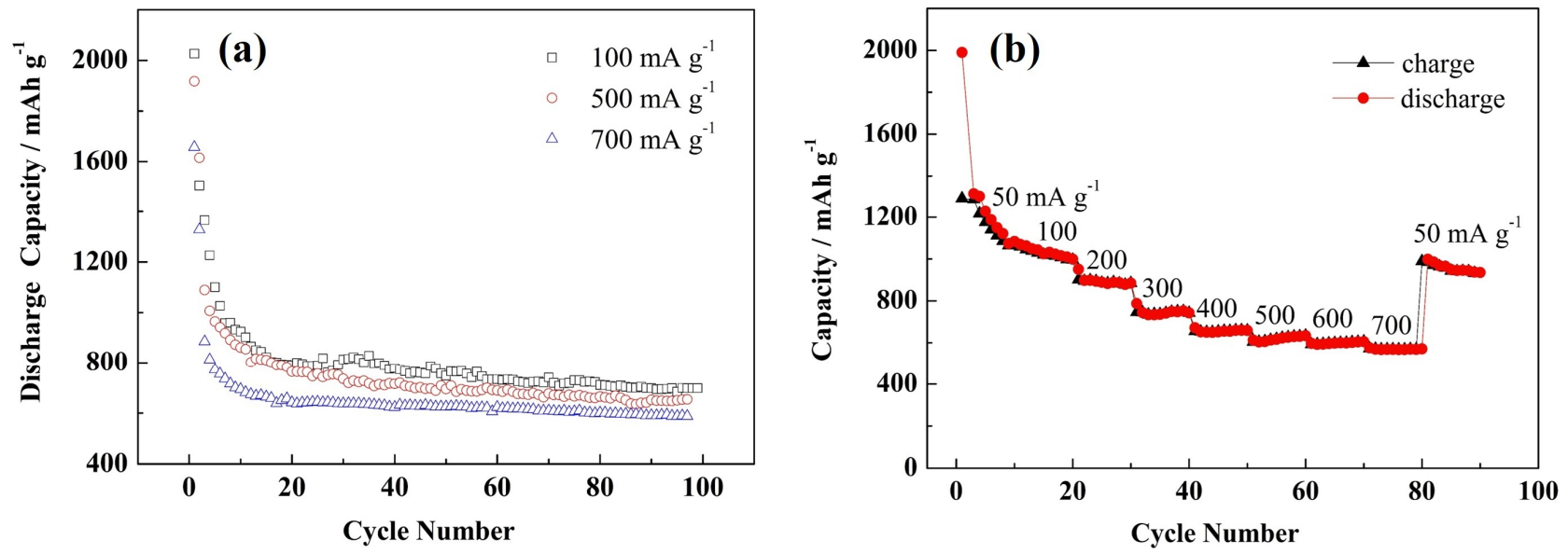

Fig. 4. (a) Cycling performances of $\mathrm{SnO}_{2}$ /graphene at 100,500 and $700 \mathrm{~mA} \mathrm{~g}^{-1}$; (b) Rate capability of $\mathrm{SnO}_{2} /$ graphene at various current densities from 100 to $700 \mathrm{~mA} \mathrm{~g}^{-1}$ 


\section{Conclusions}

$\mathrm{SnO}_{2}$ /graphene composite has been synthesized successfully by a one-step hydrothermal method. The preparation method is more simple and controllable. The $\mathrm{SnO}_{2}$ /graphene composite electrode exhibites excellent cycling performances and good rate capability in LIBs. The discharge specific capacity remains 698, 654 and $587 \mathrm{~mA} \mathrm{~h} \mathrm{~g}^{-1}$ at current densities of 100, 500 and $700 \mathrm{~mA} \mathrm{~g}^{-1}$ after 100 cycles, respectively. The excellent electr ${ }^{\circ} \mathrm{Chemical}$ performances of $\mathrm{SnO}_{2} /$ graphene composite electrode are attributed to the synergy of the monodisperse $\mathrm{SnO}_{2}$ and the high specific capacity of graphene sheets.

\section{Acknowledgements}

The work is supported by Science and Technology Research Project of the Heilongjiang Province Department of Education (no. 12531587).

\section{References}

[1] V. Etacheri, R. Marom, R. Elazari, et al, Challenges in the development of advanced Li-ion batteries: a review, Energy Environ. Sci. 4 (2011) 3243-3262.

[2] Wu Z, Zhou G, Yin L, et al, Graphene/metal oxide composite electrode materials for energy storage, Nano Energy 1 (2012) 107-131.

[3] W. Zhang, A review of the electr ${ }^{\circ}$ Chemical performance of alloy anodes for lithium-ion batteries, J. Power Sources 196 (2010) 13-24.

[4] J. Chen, X. Lou, $\mathrm{SnO}_{2}$-based nanomaterials: synthesis and application in lithium-ion batteries, Small 9 (2013) 1877-1893.

[5] P. Guo, H. Song, X. Chen, Electr ${ }^{\circ}$ Chemical performance of graphene nanosheets as anode material for lithium-ion batteries, Electr ${ }^{\circ}$ Chem. Commun. 11 (2009) 1320-1324.

[6] H. Liu, J. Huang, X. Li, et al, Hydrothermal synthesis and characterization of graphene/ self-assembled $\mathrm{SnO}_{2}$ hybrid, Physica E 44 (2012) 1931-1935.

[7] H. Liu, J. Huang, $\mathrm{X}$. Li, et al, $\mathrm{SnO}_{2}$ nanorods grown on graphite as a high-capacity anode material for lithium ion batteries, Ceramics International 38 (2012) 5145-5149.

[8] X. Zhou, Y. Yin, L. Wan, et al, A robust composite of $\mathrm{SnO}_{2}$ hollow nanospheres enwrapped by graphene as a high-capacity anode material for lithium-ion batteries, J. Mater. Chem. 22 (2012) 17456-17459.

[9] J. Zhang, X. Liu, L. Wang, et al, A simple one-pot strategy for the synthesis of ternary reduced graphite oxide/ $\mathrm{SnO}_{2} / \mathrm{Au}$ hybrid nanomaterials, Carbon 49 (2011) 3538-3543.

[10]B. Zhang, Q. Zheng, Z. Huang, et al, $\mathrm{SnO}_{2}$-graphene-carbon nanotube mixture for anode material with improved rate capacities, Carbon 49 (2011) 4524-4534.

[11]Y. Yang, R. Pang, X. Zhou, et al, Composites of chemically-reduced graphene oxide sheets and carbon nanospheres with three-dimensional network structure as anode materials for lithium ion batteries, J. Mater. Chem. 22 (2012) 23194-23200.

[12]L. Liu, M. An, P. Yang, J. Zhang, Few-layer graphene prepared via microwave digestion reduction and its electr ${ }^{\circ}$ Chemical performances in lithium ion batteries, Int. J. Elect ${ }^{\circ}$ Chem. Sci. 10 (2015) 1582-1594.

[13]A. Yang, Y. Xue, Y. Zhang, et al, A simple one-pot synthesis of graphene nanosheet $/ \mathrm{SnO}_{2}$ nanoparticle hybrid nan ${ }^{\circ}$ Composites and their application for selective and sensitive electr ${ }^{\circ}$ Chemical detection of dopamine, J. Mater. Chem. B 1 (2013) 1804-1811.

[14]D. Wang, X. Li, J. Wang, et al, Defect-rich crystalline $\mathrm{SnO}_{2}$ immobilized on graphene nanosheets with enhanced cycle performance for Li ion batteries, J. Phys. Chem. C 116 (2012) 22149-22156. 